Élodie Béthoux, Guillaume Desage, Arnaud Mias, Jérôme Pélisse, Sociologie d'un syndicalisme catégoriel. La CFE-CGC ou la fin d'une exception?

Paris, Armand Colin, coll. « Recherches », 2013

\title{
Sébastien Petit
}

\section{OpenEdition}

Journals

Édition électronique

URL : http://journals.openedition.org/travailemploi/6676

DOI : 10.4000/travailemploi.6676

ISSN : $1775-416 \mathrm{X}$

Éditeur

DARES - Ministère du Travail

Édition imprimée

Date de publication : 1 avril 2015

Pagination : 106-108

ISSN : 0224-4365

Référence électronique

Sébastien Petit, «Élodie Béthoux, Guillaume Desage, Arnaud Mias, Jérôme Pélisse, Sociologie d'un syndicalisme catégoriel. La CFE-CGC ou la fin d'une exception?», Travail et Emploi [En ligne], 142 I avriljuin 2015, mis en ligne le 01 avril 2015, consulté le 25 septembre 2020. URL : http://

journals.openedition.org/travailemploi/6676 ; DOI : https://doi.org/10.4000/travailemploi.6676

Ce document a été généré automatiquement le 25 septembre 2020.

(C) Direction de l'animation de la recherche, des études et des statistiques (Dares) 


\title{
Élodie Béthoux, Guillaume Desage, Arnaud Mias, Jérôme Pélisse, Sociologie d'un syndicalisme catégoriel. La CFE-CGC ou la fin d'une exception?
}

Paris, Armand Colin, coll. « Recherches », 2013

\author{
Sébastien Petit
}

\section{RÉFÉRENCE}

Élodie Béthoux, Guillaume Desage, Arnaud Mias, Jérôme Pélisse, Sociologie d'un syndicalisme catégoriel. La CFE-CGC ou la fin d'une exception ?, Paris, Armand Colin, coll. » Recherches », 2013, 240 p.

1 Par leur statut ${ }^{1}$ et la place qu'ils occupent dans la division du travail ${ }^{2}$, les cadres constituent, en France, une catégorie spécifique. Leur représentation et leur positionnement dans l'espace social appellent dès lors des questions qu'Élodie Béthoux, Guillaume Desage, Arnaud Mias et Jérôme Pélisse soumettent à examen en étudiant plus particulièrement le sens et la portée du syndicalisme catégoriel face aux évolutions professionnelles et sociétales récentes.

2 Les auteurs, sociologues des professions et de l'action syndicale, proposent une étude des militants syndicaux de la $\mathrm{CFE}-\mathrm{CGC}^{3}$ en les confrontant à la situation des cadres en tant que groupe. Examinant les parcours et les motivations de délégués de la CFE-CGC, ils interrogent plus largement ce qui anime le syndicalisme catégoriel, qui représentent les syndicats, quelles sont les fonctions des militants, leurs ressources et leurs pratiques, dans un contexte où la CFE-CGC est régulièrement annoncée en déclin en raison de la baisse de ses effectifs alors que le syndicalisme des cadres tend à se renforcer. 
3 Les auteurs s'appuient sur plusieurs types de matériaux: des données d'observation recueillies lors d'un congrès de la CFE-CGC ainsi qu'à l'occasion de séminaires organisés par des instances territoriales du syndicat, les résultats d'un questionnaire mené auprès des participants de ce congrès et, enfin, des entretiens réalisés avec des militants dans des unions locales et des fédérations. À travers un travail en partie sociographique, ils cherchent à mettre en exergue les principales caractéristiques de ces militants ainsi que leurs perceptions du syndicalisme chez les cadres. L'ouvrage articule, en trois temps, les représentations des militants de la CFE-CGC avec les transformations et le devenir d'une structure qui incarne, sans doute plus que d'autres, un syndicalisme en quête d'identité face aux évolutions salariales récentes.

Dans une première partie, les auteurs inscrivent la CFE-CGC dans une dimension sociohistorique. Ils rappellent ainsi que la catégorie des cadres est institutionnalisée dans les années 1930 dans un contexte fortement corporatiste. À ses débuts en 1944, né du regroupement de plusieurs centrales de représentation des cadres, le syndicat, alors appelé Confédération générale des cadres, porte trois principales revendications: le refus de la fusion avec le régime général de protection sociale, la défense d'avantages fiscaux pour les cadres et le maintien d'une hiérarchie salariale institutionnalisée. Comme l'a montré Luc Boltanski ${ }^{4}$, la CFE-CGC est longtemps apparue comme un porteparole des cadres et des ingénieurs alors que, selon Guy Groux ${ }^{5}$, elle incarne le modèle d'un syndicalisme catégoriel, corporatiste, jouant sur la différenciation avec les autres salariés.

5 Affichant une relative stabilité jusque dans les années 1960, elle entend défendre une «classe moyenne » en pleine recomposition. À partir de cette période, un débat sur la réforme de l'entreprise et sur la formation des cadres à la gestion émerge alors que les positions conservatrices du syndicat sont de plus en plus contestées par ses membres.

6 En 1981, la CFE-CGC prend sa dénomination actuelle, manifestant ainsi la volonté de s'étendre aux techniciens et aux agents de maîtrise. Les effectifs croissent mais les orientations du syndicat sont, selon les auteurs, peu marquantes et ne traduisent pas de revendications fortes. Enfin, dans un contexte d'incertitude identitaire et de déclin du syndicalisme catégoriel, la CFE-CGC adopte un ton plus revendicatif dans les années 2000 alors que la situation salariale des cadres apparaît plus instable (réduction du temps de travail, réformes des retraites et de l'assurance-chômage).

7 À la suite de ce repositionnement récent, les auteurs s'intéressent aux profils des participants d'un congrès de la CFE-CGC en 2010, dont ils entendent brosser le portrait en s'attachant à leur identité, leurs intérêts, leurs trajectoires et leurs relations. Deux lignes de clivage sont mises en avant: d'une part, une forte différenciation sectorielle; et, de l'autre, une distinction marquée entre les cadres et les non-cadres, ces derniers étant sans surprise moins attachés à la dimension catégorielle. L'élection professionnelle semble également être un facteur décisif de l'engagement syndical puisqu'elle est l'occasion de montrer le poids du syndicat et de recruter de nouveaux militants. Les directions d'entreprise et les supérieurs hiérarchiques jouent par ailleurs un rôle dans le processus d'adhésion, ce qui est propre à la syndicalisation des cadres : en effet, ils cherchent à canaliser les critiques des cadres et à relayer auprès d'eux la politique salariale de l'entreprise et d'éventuels changements organisationnels ou professionnels. 

syndicalisme en leur proposant toute une gamme de services tels que l'offre de conseils en termes de formation, de carrière, de bilan de compétences ou de recherche d'emploi. Les auteurs soulignent que la fourniture de services constitue une réponse à l'individualisme des salariés en attente de contreparties tangibles dans la foulée de leur adhésion. S'étant placés dans une logique d'offre, les syndicats sont donc contraints d'apporter au sein des entreprises des réponses aux comportements opportunistes de leurs adhérents.

ier champ d'action des syndiqués: les revendications salariales. La plupart des militants ont cherché, en adhérant, à trouver un mode d'action et de compromis pour résoudre, "sans dogme ", les problèmes qu'ils rencontrent dans leur entreprise - le terme de «compromis" revient d'ailleurs fréquemment dans les dialogues et négociations. Aussi, les revendications des militants portent d'abord sur les conditions de travail, les salaires, l'emploi et les licenciements. Allant jusqu'à la grève lorsqu'elle n'est pas reconnue comme partenaire privilégié des directions dans les négociations, la CFE-CGC n'hésite pas à médiatiser ses luttes, notamment lors des restructurations et des conflits salariaux, et entend participer à la stratégie des entreprises dans l'ensemble des secteurs économiques.

Le déploiement de la CFE-CGC dans ces différentes gammes d'actions incite les auteurs à interroger sa relation aux autres syndicats. La CFE-CGC cultive sa stratégie de différenciation sur deux registres principaux: d'une part, elle joue sur l'identité catégorielle, de l'autre, elle n'hésite pas à entrer en concurrence avec les autres syndicats. Dans le premier cas, le syndicalisme est perçu comme devant être scindé en deux mondes distincts: celui des travailleurs manuels et celui des travailleurs intellectuels. Dans le second, elle considère les autres organisations syndicales comme 
des concurrentes et leur reproche parfois de ne pas valoriser le mérite individuel. Dans les deux cas, les auteurs remarquent que la CFE-CGC affiche un attachement renouvelé aux principes de différenciation et de distinction hiérarchique - auquel le statut de cadre n'est pas étranger ${ }^{6}$.

Dans une dernière partie, les auteurs questionnent le devenir de la CFE-CGC alors que l'exception catégorielle est contestée par les autres syndicats. Selon eux, la CFE-CGC est toujours tiraillée entre défense de la singularité et désir de normalisation des rapports professionnels; de même, elle est partagée entre une position attentiste qui n'appréhende pas le syndicat comme menacé et une autre qui craint la transformation de la CFE-CGC en un syndicat généraliste.

Un principe reste prédominant dans le positionnement de la CFE-CGC, celui d'apparaitre comme un syndicat « responsable ». Pour les auteurs, il s'agit d'un possible axe de développement du syndicat; dans cette perspective, ils évoquent l'hypothèse du passage d'un syndicalisme catégoriel à un syndicalisme responsable, ce qui correspondrait, selon eux, à la transition du modèle de la qualification vers celui de la compétence. Ce tout dernier point, qui renvoie aux évolutions récentes de la division du travail, des rapports de production et de la structure de l'emploi, mériterait d'être davantage approfondi afin de préciser le rôle des cadres et de leurs représentants dans l'avènement du modèle de la compétence, lequel aboutit, notamment, à la naturalisation et à l'universalisation des normes managériales et gestionnaires.

Dans leur ouvrage, É. Béthoux, G. Desage, A. Mias et J. Pélisse ont cherché à caractériser les tensions qui structurent l'identité, les motivations et les registres d'action des délégués et militants de la CFE-CGC. Ils ont le mérite de mettre en lumière les ambivalences - sinon les contradictions - qui traversent aujourd'hui le syndicalisme catégoriel et, à travers celui-ci, le groupe des cadres lui-même. Alors qu'elle oscille entre différenciation et normalisation au sein du salariat, la CFE-CGC peine à donner une image cohérente : la définition du groupe représenté - les cadres - est toujours assortie d'un certain flou et la " classe moyenne », cible du syndicat, a des contours tout aussi incertains.

17 C'est ainsi tout un pan de recherches consacrées au syndicalisme des cadres lors du tournant managérial des années $1980^{7}$ qui est actualisé. Au-delà du rôle de relais des stratégies patronales que semblaient alors endosser les cadres, les auteurs montrent les nouvelles préoccupations des catégories d'encadrement (en termes d'emploi, de droits, de salaire, de carrière, etc.). Ils interrogent aussi les conditions de leur action collective, compte tenu du rôle qu'ils jouent dans les politiques de mobilisation des salariés mises en place par les directions d'entreprise.

Les conclusions obtenues incitent à poursuivre l'étude dans cette voie, qui peut ouvrir, notamment, sur une confrontation des incertitudes qui entourent les populations de l'encadrement et leurs représentants aux mutations du salariat et rapports sociaux dans la société contemporaine. 


\section{NOTES}

1. Boltanski L. (1982), Les cadres. La formation d'un groupe social, Paris, Éditions de Minuit ; Grelon A. (1986) (dir.), Les ingénieurs de la crise. Titre et profession entre les deux guerres, Paris, Éditions de l'École des hautes études en sciences sociales.

2. Bihr A. (1989), Entre bourgeoisie et prolétariat. L'encadrement capitaliste, Paris, L'Harmattan; Bouffartigue P., Gadéa C. (2000), Sociologie des cadres, Paris, La Découverte.

3. Confédération française de l'encadrement-Confédération générale des cadres.

4. Boltanski L., op. cit.

5. Groux G. (1983), Les cadres, Paris, Maspero.

6. Bouffartigue P. (2001), Les cadres. La fin d'une figure sociale?, Paris, La Dispute.

7. Groux G. (1983), op. cit.; Mouriaux R. (1986), Le syndicalisme face à la crise, Paris, La Découverte.

\section{AUTEURS}

\section{SÉBASTIEN PETIT}

Centre Pierre-Naville, université d'Évry 